\title{
Radiopurity of an archeological Roman Lead cryogenic detector
}

\author{
L. Pattavina ${ }^{1,2, a, b}$, J.W. Beeman ${ }^{3}$, M. Clemenza ${ }^{4,5}$, O. Cremonesi ${ }^{4}$, \\ E. Fiorini $^{4,5}$, L. Pagnanini ${ }^{4,5}$, S. Pirro ${ }^{6}$, C. Rusconi ${ }^{6,7}$, K. Schäffner ${ }^{1,6}$ \\ ${ }^{1}$ Gran Sasso Science Institute, I-67100 L'Aquila - Italy \\ ${ }^{2}$ Physik Department, Technische Universität München, D-85748 Garching - Germany \\ ${ }^{3}$ Lawrence Berkeley National Laboratory, CA-94720 Berkeley - USA \\ ${ }^{4}$ INFN - Sezione di Milano - Bicocca, I-20126 Milano - Italy \\ ${ }^{5}$ Dipartimento di Fisica, Università di Milano - Bicocca \\ ${ }^{6}$ INFN - Laboratori Nazionali del Gran Sasso, I-67100 Assergi (L'Aquila) - Italy \\ ${ }^{7}$ Department of Physics and Astronomy, University of South Carolina, SC-29208 Columbia - USA \\ Received: date / Accepted: date
}

\begin{abstract}
Archeological Roman lead $(\mathrm{Pb})$ is known to be a suitable material for shielding experimental apparata in rare event searches. In the past years the intrinsic radiopurity of this material was investigated using different technologies. In this work we applied the latest advancements in cryogenic techniques to study the bulk radiopurity of a $1 \mathrm{~cm}^{3}$ sample of archeological Roman $\mathrm{Pb}$. We report the lowest ever measured limit on ${ }^{210} \mathrm{~Pb}$ content in Roman $\mathrm{Pb}$, with a concentration lower than $715 \mu \mathrm{Bq} / \mathrm{kg}$. Furthermore, we also studied ${ }^{238} \mathrm{U}$ and ${ }^{232} \mathrm{Th}$ impurity concentrations. Our values concur with independent measurements reported in literature.
\end{abstract}

\section{Introduction}

The successful realization of ultra-low background experiments searching for elusive natural processes, such as neutrinoless double-beta decay $(0 \nu \beta \beta)$ [1], dark matternucleus [2] interactions or neutrino-nucleus interactions [3] is particularly difficult due to environmental radioactivity. Unavoidably, natural radioactivity is included in all materials employed for the detector construction, making it difficult to achieve ultra-low background conditions. As an example, if massive shielding parts surrounding the detector are not made from radiopure materials, they can become themselves the main background source, enlarging the contribution with respect to the external component (i.e. cosmic rays or high energy $\gamma \mathrm{s}$ ). For these reasons material assay is of paramount importance for low counting rate experiments.

\footnotetext{
aalso at INFN - Laboratori Nazionali del Gran Sasso

${ }^{\mathrm{b}}$ Corresponding author: luca.pattavina@lngs.infn.it
}

The first strategy adopted by ultra-low background experiments is to install their detectors in deep underground laboratories, where they can profit from the overburden rock acting as a $4 \pi$ shielding to suppress the cosmic ray induced component of the background. However, the environmental radioactivity can still limit the detector sensitivity. For this reason, further shields are installed around the experimental set-up and, depending on the size of the detector, different types of materials are employed. Large volume detectors, such as Borexino [4], Gerda [5], DarkSide [6], take advantage of the availability of highly-pure liquids (e.g. water or Ar) for shielding the detectors. At the same time they instrument the shield to function as veto detector. On the other hand, small volume detectors, such as CUORE [7], CUPID [8], CRESST [9], which require a cryogenic facility, are designed to be compact and to be shielded with an overall reduced occupancy, given the technical limitations of the cryogenic systems. It is for this reason that $\mathrm{Pb}$ is used as primary shield and it is installed in proximity of the detector's sensitive components.

$\mathrm{Pb}$ is also employed as target material for the study of neutrino properties. The two most relevant experiments are: OPERA [10], which studied $\nu_{\mu}$ oscillation in matter using $\mathrm{Pb}$ and nuclear emulsions as the core part of the detector; and HALO [11] which aims at detecting neutrinos from Supernova explosions in our galaxy using a detector made of $\mathrm{Pb}$ and ${ }^{3} \mathrm{He}$ neutron counters.

The search for highly-pure $\mathrm{Pb}$ is, therefore, a topic of interest since many years in the field of low background experiments. While the purification of liquids or gases is a well established technology [12], $\mathrm{Pb}$ purification is a complex process, due to the natural oc- 


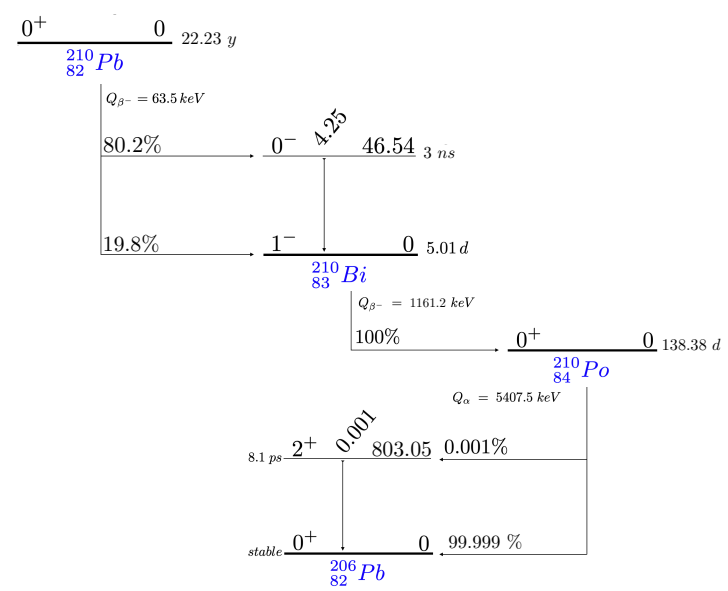

Fig. 1 Decay scheme of the ${ }^{210} \mathrm{~Pb}$ chain, adapted from [13, 14]. For ${ }^{210} \mathrm{~Pb}$ and ${ }^{210} \mathrm{Bi}$ only the $\beta^{-}$branches are shown.

currence of ${ }^{210} \mathrm{~Pb}$, which is a widely spread radioactive contaminant.

The most abundant contaminant measurable in $\mathrm{Pb}$ samples is ${ }^{210} \mathrm{~Pb}$, produced by the ${ }^{238} \mathrm{U}$ decay chain. The reduction of the concentration of ${ }^{210} \mathrm{~Pb}$ in $\mathrm{Pb}$ samples is difficult due to their affinity. Whenever a ${ }^{210} \mathrm{~Pb}$ contamination of a $\mathrm{Pb}$ sample occurs, most probably this could only be reduced by a cool-down on the time scale of the half-life of the nuclide. Therefore, it can be easily understood that ancient $\mathrm{Pb}$ samples could feature an extremely low ${ }^{210} \mathrm{~Pb}$ concentration, depending on its initial concentration.

In this work we review the status of different measurements on the radioactivity of ancient Roman Lead samples and we present the results on the excellent radiopurity of a pure $\mathrm{Pb}$ cryogenic detector made from archeological Roman $\mathrm{Pb}$.

\section{Archeological $\mathrm{Pb}$}

Archeological $\mathrm{Pb}$ is a superior raw material for shielding realization. Even though it was made some time ago its application could still be problematic due to the possible presence of ${ }^{210} \mathrm{~Pb}$.

${ }^{210} \mathrm{~Pb}$ can induce different type of signatures in a detector, its decay scheme is shown in Fig. 1. It decays through a $\beta^{-}$channel with an energy transition of $63 \mathrm{keV}$; it could populate an excited level at $46 \mathrm{keV}$ which would then produce a $46 \mathrm{keV} \gamma$ before reaching the ground state of ${ }^{210} \mathrm{Bi}$. This nuclide has a short halflife $(5 \mathrm{~d})$, and it decays a through $\beta^{-}$channel releasing $1.16 \mathrm{MeV}$ of energy shared among the decay products. The energetic electron can produce bremsstrahlung radiation but also high-energy X-rays (up to $90 \mathrm{keV}$ ), while crossing the $\mathrm{Pb}$ sample.
After its decay, ${ }^{210} \mathrm{Bi}$ populates the ground state of ${ }^{210} \mathrm{Po}$ which, and in most of the cases (99.99\% [15]) $\alpha$ decays on the stable nuclide ${ }^{206} \mathrm{~Pb}$. The energy of the $\alpha$ is about $5.3 \mathrm{MeV}$, and it is considered a relevant source of background for $0 \nu \beta \beta$ experiments [16]. In order to mitigate this possible background source, $\mathrm{Pb}$ is never directly facing cryogenic detectors but a thick layer of highly-pure $\mathrm{Cu}$ is always interleaved between the two.

While studying the radiopurity of modern $\mathrm{Pb}$ samples and $\mathrm{Pb}$-based compounds, high levels of ${ }^{210} \mathrm{~Pb}$ concentration can be measured $[17,18]$ at the level of tens to hundreds of $\mathrm{Bq} / \mathrm{kg}$. This is due to the presence of ${ }^{238} \mathrm{U}$ decay chain products in $\mathrm{Pb}$ ores, as ${ }^{210} \mathrm{~Pb}$. In fact, when the ${ }^{238} \mathrm{U}$ decay chain is in secular equilibrium ${ }^{1}$, then ${ }^{210} \mathrm{~Pb}$ is continuously produced from the successive decays of ${ }^{238} \mathrm{U}$ and its daughter nuclides.

While refining raw metal $\mathrm{Pb}$, a ${ }^{210} \mathrm{~Pb}$ concentration process is encountered, as a matter of fact. In fact, while other radioactive nuclides which are chemically not affine to $\mathrm{Pb}$ are segregated from the slag, ${ }^{210} \mathrm{~Pb}$ is concentrated. This process directly results in high purity $\mathrm{Pb}$, where a high concentration of ${ }^{210} \mathrm{~Pb}$ could be present. As already discussed, a cool-down time sufficiently long would allow to reduce this residual radioactivity, making $\mathrm{Pb}$ an excellent highly-pure material for low background physics. Within the Roman Empire age, $\mathrm{Pb}$ was refined for various purposes [19] providing nowadays an important unintentional source of low radioactivity $\mathrm{Pb}$.

The radiopurity of ancient Roman $\mathrm{Pb}$ was widely investigated and very low levels of intrinsic contaminations were found. The current best value for ${ }^{210} \mathrm{~Pb}$ is a limit of $4 \mathrm{mBq} / \mathrm{kg}$ [20], which was obtained using an absorber of pure $\mathrm{Pb}$ operated as cryogenic detector. This innovative technique was proposed because of the limited sensitivity of previous measurements. The standard technique used up to the publication time of [20] was $\gamma$-spectrometry with HP-Ge detectors, which provided limits at the level of hundreds of $\mathrm{mBq} / \mathrm{kg}[22]$. The HP-Ge (High-Purity Germanium) technique is not as sensitive as the cryogenic technique, because of the extremely low detection efficiency due both to geometrical reasons (the detector and the sample are separated) and to self-absorption in the sample. In fact, the most common approach is to detect the $46 \mathrm{keV} \gamma$ which is mostly absorbed by the sample itself. Moreover the emission has a very small branching ratio of $4 \%$ (see Fig. 1). Instead, the cryogenic technique studies ${ }^{210} \mathrm{Po}$, the decay product ${ }^{210} \mathrm{~Pb}$. The signal induced by ${ }^{210} \mathrm{Po}$ is in a favourable region of the energy spectrum with an excellent signal-to-background ratio (at $5.4 \mathrm{MeV}$ ), and it also has a branching ratio of almost $100 \%$.

${ }^{1}$ All the nuclides of the decay chain have the same activity. 


\begin{tabular}{|c|c|c|c|}
\hline Detector & ${ }^{210} \mathrm{~Pb}$ & Sample mass & Ref. \\
\hline & {$[\mathrm{mBq} / \mathrm{kg}]$} & {$[\mathrm{kg}]$} & \\
\hline HP-Ge & $<900$ & 4.5 & {$[22]$} \\
\hline HP-Ge & $<1300$ & 22.1 & {$[23]$} \\
\hline Planar Si & 100 & 0.01 & {$[24]$} \\
\hline Bolometer & $<20$ & 0.09 & {$[25]$} \\
\hline Bolometer & $<4$ & 0.01 & {$[20]$} \\
\hline
\end{tabular}

Table 1 Summary of the most sensitive measurements on the concentration of ${ }^{210} \mathrm{~Pb}$ in ancient $\mathrm{Pb}$ samples using different techniques.

Spectrometry with HP-Ge detectors is extremely effective for investigating the concentration of ${ }^{238} \mathrm{U}$ and ${ }^{232} \mathrm{Th}$ decay chain products. This is possible thanks to the high energy $\gamma \mathrm{s}$ produced by these nuclides, (up to $2.6 \mathrm{MeV}$ ) which reduce the possibility of self-absorption in the sample, therefore enhancing the detection efficiency. This very same technique, as already discussed, suffers from extremely low detection efficiency for low energy $\gamma$ lines (e.g. ${ }^{210} \mathrm{~Pb} \gamma$ emission).

Currently the best limits on the concentration of ${ }^{238} \mathrm{U}$ and ${ }^{232} \mathrm{Th}$ decay chain products are: $<46 \mu \mathrm{Bq} / \mathrm{kg}$ and $<45 \mu \mathrm{Bq} / \mathrm{kg}$, respectively [21]. These values are true under the hypothesis that all the elements of the decay chains have the secular equilibrium established in all the parts of the radioactive chain. This is a rather strong assumption given that, depending on how the sample is handled and produced, secular equilibrium could be broken due to the different chemical properties of the decay products.

In Tab. 1 a summary of the most relevant measurements on low-activity $\mathrm{Pb}$ samples is shown.

\section{Archeological $\mathrm{Pb}$ based detectors}

Many works can be found in literature where ancient $\mathrm{Pb}$ is embedded in cryogenic particle detectors. We can identify two classes of detectors: Pb-based scintillators, such as $\mathrm{PbWO}_{4}$ [26] or $\mathrm{PbMoO}_{4}$ [27], and pure-Pb absorbers [20].

The possibility to produce detectors, namely scintillating crystals, from radiopure materials is paramount. In fact, a detector with excellent energy resolution and with the ability to perform particle identification can enhance the physics potential of an experiment. $\mathrm{Pb}-$ based crystals are known to be excellent scintillators, but unfortunately they suffer from an excess of radioactivity that makes them not suitable for rare event investigations. The reason is the high concentration of ${ }^{210} \mathrm{~Pb}$ which enters in the crystal during its production/growth. For the growth of commercial Pb-based

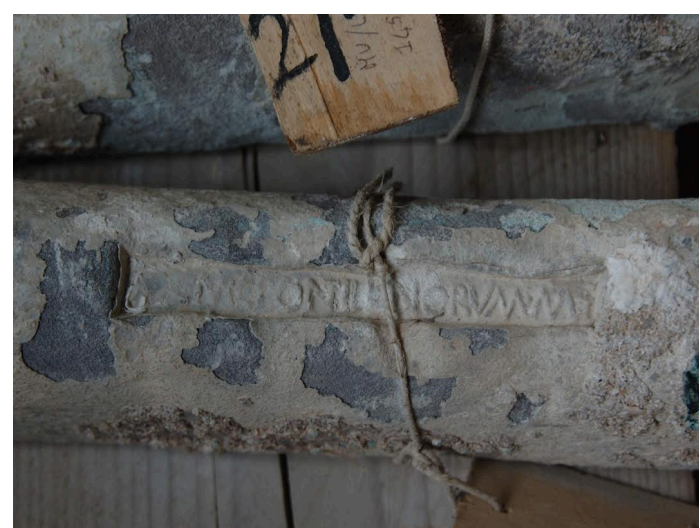

Fig. 2 Brick of ancient Roman $\mathrm{Pb}$ found in the Mediterranean see off the Sardinia shores. The ingot is has a mass of about $23 \mathrm{~kg}$ and it is mostly covered by sea salts residuals.

crystals, modern $\mathrm{Pb}$ is used, known to feature a high ${ }^{210} \mathrm{~Pb}$ concentration at the level of $\mathrm{Bq} / \mathrm{kg}$. Furthermore, such commercial crystals could also exhibit high concentrations of ${ }^{238} \mathrm{U}$ and ${ }^{232} \mathrm{Th}$ decay chain products.

Recently, this problem was overcome by the use of ancient Roman $\mathrm{Pb}$ for the production of scintillating crystals such as $\mathrm{PbWO}_{4}$ and $\mathrm{PbMoO}_{4}$. Regrettably, these compounds still show a high concentration of ${ }^{210} \mathrm{~Pb}$, ${ }^{238} \mathrm{U}$ and ${ }^{232} \mathrm{Th}$ at the level of hundreds of $\mathrm{mBq} / \mathrm{kg}$, due to a not complete control of the crystal growth process.

In 1998, pure $\mathrm{Pb}$ detectors were also successfully operated [20] and featured excellent radiopurity levels, thanks to the absence of any production process. The samples were directly cut from the bulk of an ancient Roman $\mathrm{Pb}$ brick as the one shown in Fig. 2.

These samples (10 $\mathrm{g}$ of mass each) were equipped with a Ge Neutron Transmutation Doped (Ge-NTD) thermal sensor and operated as cryogenic detectors at about $10 \mathrm{mK}$. The achieved energy resolution was in the range of $100 \mathrm{keV} \mathrm{FWHM} \mathrm{at} 1.3 \mathrm{MeV}$, and, while investigating the ${ }^{210} \mathrm{~Pb}$ content in the detector, no ${ }^{210} \mathrm{Po}$ signal above the background level was observed. A limit on the ${ }^{210} \mathrm{~Pb}$ concentration in the detector was set $<4 \mathrm{mBq} / \mathrm{kg}[20]$ at $95 \%$ C.L.

The difficulties in running such detectors are highlighted by the poor energy resolution. In fact operating a cryogenic detector in a superconducting state ${ }^{2}$ is not trivial when using a thermal sensor sensitive to thermal phonons, like a Ge-NTD. Furthermore the Debye temperature of the detector is about $80 \mathrm{~K}$, which makes its heat capacity at low temperatures not favourable for low energy studies.

The performance of such detectors might be enhanced operating $\mathrm{Pb}$ with other classes of thermal sensors, suitable to read out superconducting absorber.

${ }^{2} \mathrm{~Pb}$ has a critical temperature of $7.2 \mathrm{~K}$. 


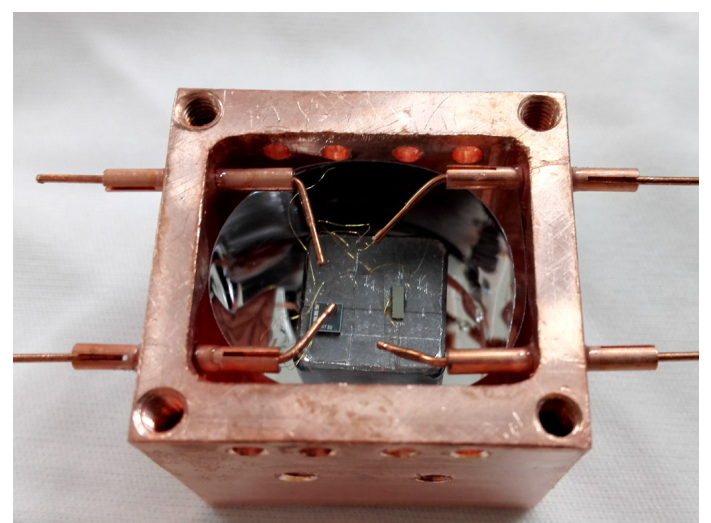

Fig. 3 Pure $\mathrm{Pb}$ cryogenic detector. The $1 \mathrm{~cm}^{3} \mathrm{~Pb}$ absorber is visible inside the $\mathrm{Cu}$ housing. The Ge-NTD thermal sensor (on the right) and the Si Joule heater (on the left) are also visible.

\section{$4 \mathrm{Pure- \textrm {Pb }}$ cryogenic detector}

The detector used for the measurement described in this work is one of the two samples already employed in [20]. The absorber has a volume of $1 \mathrm{~cm}^{3}$ and a mass of $10 \mathrm{~g}$. This was extracted from a $23 \mathrm{~kg}$ Roman $\mathrm{Pb}$ ingot, without the need of any further processing.

The absorber was equipped with a Ge-NTD thermistor similar to the one used in [28], but with reduced dimensions: $3.0 \times 1.0 \times 0.3 \mathrm{~mm}^{3}$. The choice for a miniaturized thermal sensor is driven by the possibility to enhance the detector performance, namely the signal amplitude, while reducing the overall heat capacity of the system: absorber plus thermal sensor. The detector was also instrumented with a Si heater which was operated as Joule resistor, meant for the monitoring and the correction of temperature drifts of the absorber during the data taking.

The absorber was installed in a $\mathrm{Cu}$ housing and fixed to the support structure by means of epoxy resin. Copper was chosen not only because of its excellent thermal conductance at low temperatures, but also for minimizing possible surface radioactive backgrounds, given its excellent radiopurity. Our region of interest lies around 5.4 MeV (Q-value of ${ }^{210} \mathrm{Po}$ ), thus a possible background source can be ascribed to surface $\alpha$ decays as discussed in [29]. A picture of the complete detector is shown in Fig. 3.

The detector was installed in the low background CUPID R\&D cryogenic infrastructure located at the Laboratori Nazionali del Gran Sasso of INFN (Italy).

The thermal sensor was biased with a constant voltage through two 5 G $\Omega$ load resistors in series, which ensured a constant current flow of $2.9 \mathrm{nA}$ through the thermistor. Its working resistance was $2.7 \mathrm{M} \Omega$, thus much lower than the load resistors in series. The ac- quired signals were amplified by custom-made low noise electronics [30] and then filtered by means of a 6-pole Bessel filter with cutting frequency of $500 \mathrm{~Hz}$. Finally, the signals were fed into a NI-PXI-6284 18-bit ADC. The sampling frequency was $1 \mathrm{kHz}$ and the waveforms were recorded when a software derivative trigger fired.

\section{Results}

The detector was operated for about $300 \mathrm{~h}$, during which both background and calibration data were collected. During calibration runs the detector was exposed to a ${ }^{232} \mathrm{Th}$ source deployed next to the experimental set-up, but outside the cryogenic system. Characteristic monochromatic $\gamma$ emissions from the source were used for the calibration of the energy response of the detector, as well as for the monitoring of detector stability in time. Furthermore, a low intensity ${ }^{232} \mathrm{Th}$ source was also installed nearby the detector, to study the detector response at lower energies.

The detector response to $\alpha$ interactions was not investigated in order not to spoil the detector background. In fact, this would have required the installation of a permanent $\alpha$ decay source directly facing the detector. This type of investigation was already performed with this very same detector in previous studies, in which the detector response was assumed to be the same for both $\alpha$ and $\beta / \gamma$ interactions.

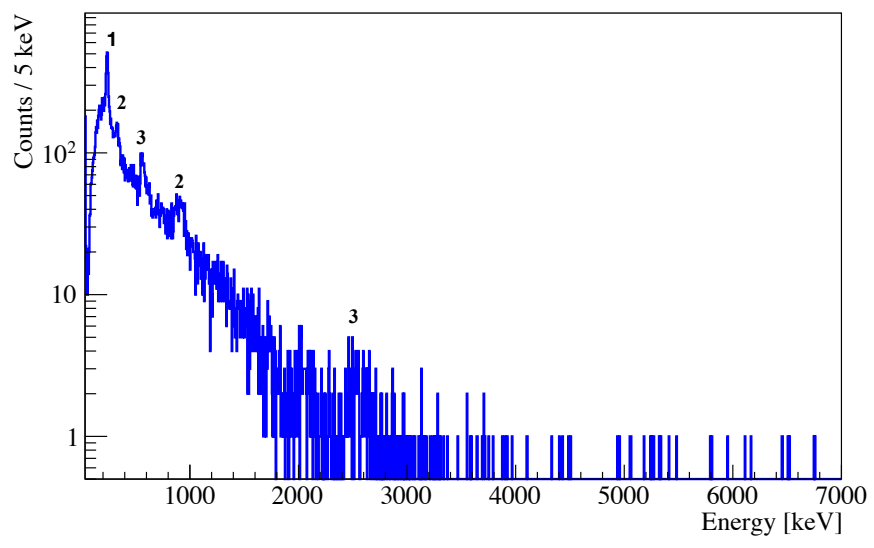

Fig. 4 Total background energy spectrum of a $1 \mathrm{~cm}^{3}$ pure $\mathrm{Pb}$ cryogenic detector acquired over $229 \mathrm{~h}$. The peaks are labeled as follows: (1) ${ }^{212} \mathrm{~Pb}$, (2) ${ }^{228} \mathrm{Ac}$, and (3) ${ }^{208} \mathrm{Tl}$.

Background data were acquired for $229 \mathrm{~h}$, when the detector was not exposed to calibration sources. The triggered data were studied by means of a two levels analysis system. Firstly, data were selected rejecting periods of noise excess due to interferences in the experimental set-up (i.e. electromagnetic disturbances or 
microphonic noise). Then, a second level data selection was performed, where by means of a pulse-shape analysis only particle events are selected. The total data selection efficiency is estimated to be $95 \pm 4 \%$ at $2.6 \mathrm{MeV}$, the most intense high energy monochromatic $\gamma$-line in the acquired energy spectrum.

The results of the data selection analysis are shown in Fig. 4, reporting the total background energy spectrum.

The energy spectrum features few monochromatic $\gamma$ lines, ascribed to the near ${ }^{232} \mathrm{Th}$ calibration source. The FWHM resolution for the most prominent line, from ${ }^{212} \mathrm{~Pb}$, at $238 \mathrm{keV}$, is $22 \pm 1 \mathrm{keV}$. At this energy scale the detector response is clearly Gaussian, as shown in Fig. 5.

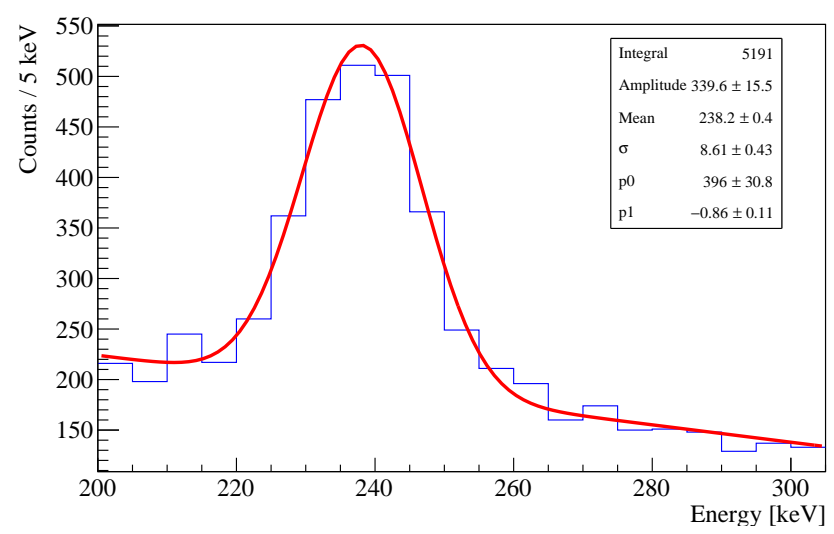

Fig. 5 Detector energy response at $238 \mathrm{keV}$. This signature is induced by a $\gamma$ interaction from ${ }^{212} \mathrm{~Pb}$. The data are fitted using a convolution of a Gaussian function with a linear background.

For the detector energy calibration and the study of the detector response at higher energies, an intense ${ }^{232} \mathrm{Th}$ source was deployed next to the cryogenic system. The detector response at the highest energy $\gamma$ line $(2.6 \mathrm{MeV})$ is shown in Fig. 6. Different line shapes were studied, but the simplest that reproduced the detector response was a double-Gaussian. The reason for such a shape may rely on the non-uniformity of the detector response. In fact the detector is not a single crystal and possible position dependence effects could influence the response at high energies, due to multi-Compton interactions in the absorber. This behaviour was also observed in [7]. The FWHM resolution at $2.6 \mathrm{MeV}$ is $103.6 \pm 9.0 \mathrm{keV}$.

As shown in Fig. 4, the detector did not feature any clear signature around $5.4 \mathrm{MeV}$, which is exactly the Q-value of ${ }^{210} \mathrm{Po}$. In first approximation we can assume that the detector energy resolution scales linearly with energy [8], for this reason we would expect the energy

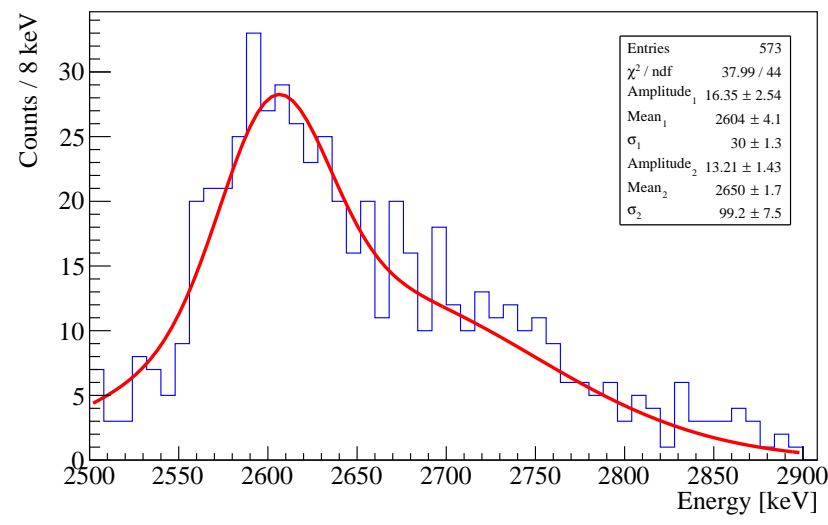

Fig. 6 Detector energy response at 2.6 MeV. This signature is induced by $\gamma$ interaction from ${ }^{208} \mathrm{Tl}$. The data are fitted using a double-Gaussian function.

resolution at $5.4 \mathrm{MeV}$ to be $200 \pm 17 \mathrm{keV}$. In Fig. 7, a zoom in around the region of interest is shown.

We determine the ${ }^{210} \mathrm{~Pb}$ activity in our sample applying the Feldman-Cousin method [31]. The signal is evaluated in the $200 \mathrm{keV}$ RoI around the Q-value (4 events), while the background on the adjacent side bands (3 events). The upper limit at a $90 \%$ C.L. is $<715 \mu \mathrm{Bq} / \mathrm{kg}$ ( 5.6 events) at a $90 \%$ confidence level. The result achieved with this set-up is about one order of magnitude better than any previous established limit. The detector counting rate in this region is extremely small, demonstrating the excellent radiopurity level of archeological Roman $\mathrm{Pb}$.

Using the same approach previously described, we were able to set upper limits on the concentration of ${ }^{232} \mathrm{Th}$ and ${ }^{238} \mathrm{U}$ decay chains, which are $<0.44 \mathrm{mBq} / \mathrm{kg}$ and $<0.34 \mathrm{mBq} / \mathrm{kg}$, respectively. These values are not competitive with the ones reported in [16], but it is a further confirmation of the purity of such valuable material.

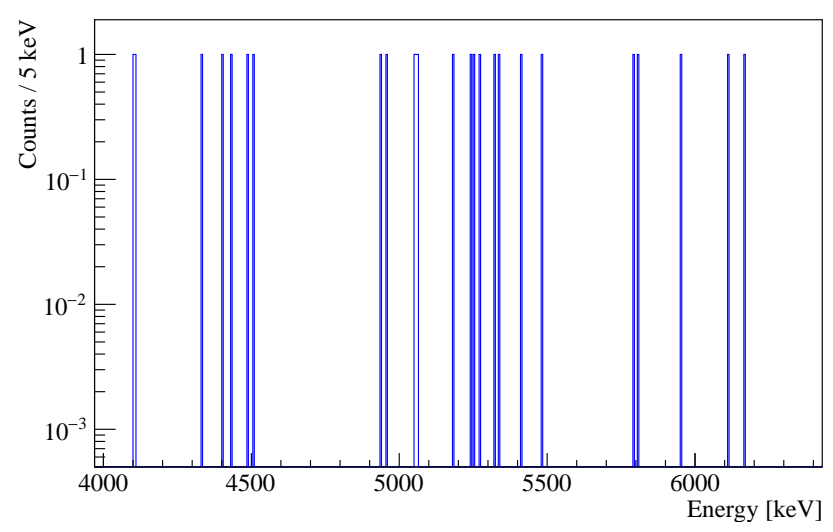

Fig. 7 Total background energy spectrum acquired over $229 \mathrm{~h}$ zoomed in the region of interest. 


\section{Conclusions}

In this work we operated a $1 \mathrm{~cm}^{3}$ cryogenic detector made of pure archeological Roman $\mathrm{Pb}$, one of the two absorbers that was used in [20]. The low-background environment in which we ran the detector allowed us to study the internal radiopurity of the absorber. The most stringent limit on the concentration of ${ }^{210} \mathrm{~Pb}$ was established to be $<715 \mu \mathrm{Bq} / \mathrm{kg}\left(<2.4 \times 10^{-19} \mathrm{~g} / \mathrm{g}\right)$. The absorber features an overall excellent radiopurity and no excess of ${ }^{232} \mathrm{Th}$ and ${ }^{238} \mathrm{U}$ decay chain products was observed; limits on their concentration were determined to be $<0.44 \mathrm{mBq} / \mathrm{kg}$ and $<0.34 \mathrm{mBq} / \mathrm{kg}$, respectively.

The excellent radiopurity level and the current performance as cryogenic detector make this material an interesting candidate for neutrino physics applications such as coherent neutrino-nucleus elastic scattering, but especially for the detection of supernova neutrinos, as discussed in [32]. The performance as a cryogenic detector can be further optimized by the growth of a singlecrystal absorber (e.g. melting and ri-crystallization of ancient $\mathrm{Pb}$ ) and by the development of suitable thermal sensors (e.g. Ge-NTDs or Transition Edge Sensors).

Acknowledgements Thanks are due to the LNGS mechanical workshop and in particular to E. Tatananni, A. Rotilio, A. Corsi, and B. Romualdi for continuous and constructive help in the overall set-up construction. Finally, we are especially grateful to M. Perego and M. Guetti for their invaluable help, and also to M. Nastasi for fruitful discussions.

\section{References}

1. J. Engel and P. Vogel, Physics 11, 30 (2018).

2. G. Bertone et al., J. Cosm. Astrop. Phys. 03, 026 (2018).

3. J.B. Dent al., Phys. Rev. D 97, 035009 (2018).

4. G. Alimonti (Borexino Coll.) et al., Nucl. Instr. Meth. A 600, 568 (2009).

5. M. Agostini et al. (GERDA Collaboration), Eur. Phys. J. C 78, 388 (2018).

6. P. Agnes et al. (Dark Side Collaboration), Phys. Rev. Lett. 121, 081307 (2018).

7. C. Alduino et al. (CUORE Collaboration), Phys. Rev. Lett. 120, 132501 (2018).

8. O. Azzolini et al., Phys. Rev. Lett. 120, 232502 (2018).

9. G. Angloher et al. (CRESST Collaboration), Eur. Phys. J. C 76, 25 (2016).

10. N. Agafonova et al. (OPERA Collaboration), J. Instrum. 4, P06020 (2009).

11. C.A. Duba et al., J. Phys.: Conf. Ser. 136, 042077 (2008).

12. M. Balata et al., Nucl. Instr. Meth. A 370, 605 (1996).

13. Atomic and Nuclear data base: http://www.lnhb.fr/nuclear-data

14. R.B. Firestone et al., Table of Isotopes, 8th Edition, Wiley-VCH Berlin (1998).

15. A. Shor et al., Phys. Rev. C 97, 034303 (2018).

16. C. Alduino, (CUORE Coll.) et al., Eur. Phys. J. C 77, 543 (2017).

17. M. Laubenstein et al., Appl. Rad. and Isotopes 61, 167 (2004).
18. K. Bunzl and W. Kracke, Nucl. Instr. Meth. A 238, 191 (1985).

19. F. Callatay, J. Roman Archaeol. 18, 361 (2005).

20. A. Alessandrello et al., Nucl. Instr. Meth. B 142, 163 (1998).

21. M. Clemenza, J. Radioanal. Nucl. Chem. 318, 1765 (2018).

22. A. Alessandrello et al., Nucl. Instr. Meth. B 61, 106 (1991).

23. G. Heusser et al., Low-level germanium?-ray spectrometry at the? Bq $/ \mathrm{kg}$ level and future developments towards higher sensitivity, in P. P. Povinec and J. A. SanchezCabeza (Eds.), Radionuclides in the Environment, Elsevier, Amsterdam (2006), pg. 495.

24. J.L. Orrell et al., J. Radioanal. Nucl. Ch. 309, 1271 (2016).

25. A. Alessandrello et al., Nucl. Instr. Meth. B 83, 539 (1993).

26. J.W. Beeman et al., Eur. Phys. J. A 49, 50 (2013).

27. S . Nagorny et al., J. Phys.: Conf. Ser. 84, 012025 (2017).

28. O. Azzolini, et al., Eur. Phys. J. C 78, 428 (2018).

29. M. Clemenza et al., Eur. Phys. J. C 71, 1805 (2011).

30. C. Arnaboldi, et al., Nucl. Instrum. Meth. A 520, 578 (2004).

31. G. Feldman, R. Cousins, Phys. Rev. D 57, 3873 (1998).

32. A. Bandyopadhyay et al. Phys. Rev. D 95, 065022 (2017). 